PROCEEDINGS OF THE

AMERICAN MATHEMATICAL SOCIETY

Volume 131, Number 2, Pages 523-531

S 0002-9939(02)06601-7

Article electronically published on June 3, 2002

\title{
IRRATIONAL ROTATION NUMBERS AND UNBOUNDEDNESS OF SOLUTIONS OF THE SECOND ORDER DIFFERENTIAL EQUATIONS WITH ASYMMETRIC NONLINEARITIES
}

\author{
ZAIHONG WANG
}

(Communicated by Carmen C. Chicone)

ABstract. In this paper, we study the dynamics of the mappings

$$
\left\{\begin{array}{l}
\theta_{1}=\theta+2 \alpha \pi+\frac{1}{r} \mu_{1}(\theta)+o\left(r^{-1}\right), \\
r_{1}=r+\mu_{2}(\theta)+o(1), \quad r \rightarrow+\infty,
\end{array}\right.
$$

where $\alpha$ is a irrational rotation number. We prove the existence of orbits that go to infinity in the future or in the past by using the well-known Birkhoff Ergodic Theorem. Applying this conclusion, we deal with the unboundedness of solutions of Liénard equations with asymmetric nonlinearities.

\section{INTRODUCTION}

We are concerned with the unboundedness of solutions of the second order differential equations

$$
x^{\prime \prime}+f(x) x^{\prime}+a x^{+}-b x^{-}=p(t),
$$

where $a$ and $b$ are positive constants, $x^{+}=\max \{x, 0\}, x^{-}=\max \{-x, 0\}, f(x)$ is a continuous function and $p(t)$ is a continuous $2 \pi$-periodic function. Throughout this paper, we define $F(x)=\int_{0}^{x} f(x) d x$ and so $F(x) \in C^{1}(\mathbb{R})$.

When $f(x) \equiv 0$, Eq. (1.1) becomes

$$
x^{\prime \prime}+a x^{+}-b x^{-}=p(t),
$$

which was first studied by Dancer in [1, 2] and Fucik in 3]. Up to now, there have appeared many results about the existence of periodic solutions and boundedness (or unboundedness) of solutions of Eq. (1.2) [4], [12]. When $a$ and $b$ are different and satisfy

$$
\frac{1}{\sqrt{a}}+\frac{1}{\sqrt{b}} \in \mathbb{Q}
$$

J. M. Alonso and R. Ortega [12] proved the existence of periodic functions $p(t)$ such that all the solutions of (1.2) with large initial conditions are unbounded. In order

Received by the editors March 19, 2001 and, in revised form, September 22, 2001.

2000 Mathematics Subject Classification. Primary 34C25; Secondary 34B15.

Key words and phrases. Unboundedness of solution, action-angle variable, asymmetric nonlinearity.

The author's research was supported by the National Natural Science Foundation of China, No.10001025, and by the Natural Science Foundation of Beijing (1022003). 
to prove the unboundedness of solutions of Eq. (1.2), they studied the dynamics of a class of mappings defined on the plane, which have an asymptotic expression

$$
\left\{\begin{array}{l}
\theta_{1}=\theta+2 \pi \frac{p}{q}+\frac{1}{r} \mu_{1}(\theta)+o\left(r^{-1}\right), \\
r_{1}=r+\mu_{2}(\theta)+o(1), \quad r \rightarrow+\infty,
\end{array}\right.
$$

where $p / q$ is a rational number and $\mu_{1}, \mu_{2}$ are continuous and $2 \pi$-periodic functions. They proved the existence of orbits that go to infinity in the future provided that there exists $\omega \in R$ such that

$$
\mu_{2}(\omega)>0, \mu_{1}(\omega)=0, \mu_{1}(\theta)(\theta-\omega)<0 \text { for } \theta \neq \omega \text { and }|\theta-\omega| \text { is small }
$$

or in the past provided that there exists $\omega \in R$ such that

$$
\mu_{2}(\omega)<0, \mu_{1}(\omega)=0, \mu_{1}(\theta)(\theta-\omega)>0 \text { for } \theta \neq \omega \text { and }|\theta-\omega| \text { is small. }
$$

In the present paper, we will study the unboundedness of solutions of Eq. (1.1) when $a$ and $b$ satisfy

$$
\frac{1}{\sqrt{a}}+\frac{1}{\sqrt{b}} \in \mathbb{R} \backslash \mathbb{Q} .
$$

Similarly, we will study the dynamics of mappings

$$
\left\{\begin{array}{l}
\theta_{1}=\theta+2 \alpha \pi+\frac{1}{r} \mu_{1}(\theta)+o\left(r^{-1}\right), \\
r_{1}=r+\mu_{2}(\theta)+o(1), \quad r \rightarrow+\infty,
\end{array}\right.
$$

where $\alpha$ is an irrational number. Under certain conditions, we prove the existence of orbits that go to infinity in the future or in the past by using the well-known Birkhoff Ergodic Theorem. On the basis of this conclusion, we obtain the following theorems.

Theorem 1. Assume that $1 / \sqrt{a}+1 / \sqrt{b} \in \mathbb{R} \backslash \mathbb{Q}$ and the limits $\lim _{x \rightarrow+\infty} F(x)=$ $F(+\infty), \lim _{x \rightarrow-\infty} F(x)=F(-\infty)$ exist and are finite. Moreover, $F(+\infty)<0<$ $F(-\infty)$. Then there exists $R_{0}>0$ such that every solution $x(t)$ of (1.1) with

$$
x\left(t_{0}\right)^{2}+x^{\prime}\left(t_{0}\right)^{2} \geq R_{0}^{2}
$$

with some $t_{0} \in \mathbb{R}$ goes to infinity in the future.

Theorem 2. Assume that $1 / \sqrt{a}+1 / \sqrt{b} \in \mathbb{R} \backslash \mathbb{Q}$ and the limits $\lim _{x \rightarrow+\infty} F(x)=$ $F(+\infty), \lim _{x \rightarrow-\infty} F(x)=F(-\infty)$ exist and are finite. Moreover, $F(-\infty)<0<$ $F(+\infty)$. Then there exists $R_{0}>0$ such that every solution $x(t)$ of (1.1) with

$$
x\left(t_{0}\right)^{2}+x^{\prime}\left(t_{0}\right)^{2} \geq R_{0}^{2}
$$

with some $t_{0} \in \mathbb{R}$ goes to infinity in the past.

\section{UNBOUNDED ORBITS OF PLANAR MAPPINGS}

Let $\sigma>0$ be a sufficiently large constant. Set

$$
E_{\sigma}=\left\{(x, y): x^{2}+y^{2} \geq \sigma^{2}\right\} .
$$

Assume that $\mathcal{P}: E_{\sigma} \rightarrow R^{2}$ is a one-to-one and continuous mapping, whose lift can be expressed in the form

$$
\left\{\begin{array}{l}
\theta_{1}=\theta+2 \alpha \pi+\frac{1}{r} \mu_{1}(\theta)+H(\theta, r), \\
r_{1}=r+\mu_{2}(\theta)+G(\theta, r)
\end{array}\right.
$$


where

$$
\alpha \in \mathbb{R} \backslash \mathbb{Q}, \quad \mu_{1}, \mu_{2}: S^{1} \rightarrow S^{1} \text { are Lipschitz continuous }, S^{1}=\mathbb{R} / 2 \pi Z
$$

and $H, G$ are $2 \pi$-periodic in $\theta$ and satisfy

$$
r|H(\theta, r)|+|G(\theta, r)| \rightarrow 0 \text { as } r \rightarrow+\infty,
$$

uniformly with respect to $\theta \in R$.

Given a point $\left(\theta_{0}, r_{0}\right)$, denote by $\left\{\left(\theta_{n}, r_{n}\right)\right\}$ the orbit of the mapping $\mathcal{P}$ through the point $\left(\theta_{0}, r_{0}\right)$. That is to say

$$
\left(\theta_{n+1}, r_{n+1}\right)=\mathcal{P}\left(\theta_{n}, r_{n}\right) .
$$

Proposition 2.1. Assume that conditions (2.2), (2.3) hold and

$$
\int_{0}^{2 \pi} \mu_{2}(\theta) d \theta>0 .
$$

Then there exists $R_{0}>\sigma$ such that if $r_{0} \geq R_{0}$, the orbit $\left\{\left(\theta_{n}, r_{n}\right)\right\}$ satisfies

$$
\lim _{n \rightarrow+\infty} r_{n}=+\infty \text {. }
$$

Proof. From the expression of the mapping $\mathcal{P}$ we have that

$$
\left\{\begin{array}{l}
\theta_{2}=\theta_{1}+2 \alpha \pi+\frac{1}{r_{1}} \mu_{1}\left(\theta_{1}\right)+H\left(\theta_{1}, r_{1}\right) \\
r_{2}=r_{1}+\mu_{2}\left(\theta_{1}\right)+G\left(\theta_{1}, r_{1}\right)
\end{array}\right.
$$

Therefore,

$$
\left\{\begin{array}{l}
\theta_{2}=\theta_{0}+4 \alpha \pi+\frac{1}{r_{0}} \mu_{1}\left(\theta_{0}\right)+H\left(\theta_{0}, r_{0}\right)+\frac{1}{r_{1}} \mu_{1}\left(\theta_{1}\right)+H\left(\theta_{1}, r_{1}\right) \\
r_{2}=r_{0}+\mu_{2}\left(\theta_{0}\right)+G\left(\theta_{0}, r_{0}\right)+\mu_{2}\left(\theta_{1}\right)+G\left(\theta_{1}, r_{1}\right)
\end{array}\right.
$$

Since

$$
\frac{1}{r_{1}}=\frac{1}{r_{0}+\mu_{2}\left(\theta_{0}\right)+G\left(\theta_{0}, r_{0}\right)}=\frac{1}{r_{0}}+O\left(\frac{1}{r_{0}^{2}}\right)
$$

and

$$
\mu_{1}\left(\theta_{1}\right)=\mu_{1}\left(\theta_{0}+2 \alpha \pi+\frac{1}{r_{0}} \mu_{1}\left(\theta_{0}\right)+H\left(\theta_{0}, r_{0}\right)\right)=\mu_{1}\left(\theta_{0}+2 \alpha \pi\right)+O\left(\frac{1}{r_{0}}\right),
$$

we know that

$$
\frac{1}{r_{1}} \mu_{1}\left(\theta_{1}\right)=\frac{1}{r_{0}} \mu_{1}\left(\theta_{0}+2 \alpha \pi\right)+O\left(\frac{1}{r_{0}^{2}}\right) .
$$

Then $\theta_{2}$ can be expressed in the form

$$
\theta_{2}=\theta_{0}+4 \alpha \pi+\frac{1}{r_{0}}\left[\mu_{1}\left(\theta_{0}\right)+\mu_{1}\left(\theta_{0}+2 \alpha \pi\right)\right]+H_{2}\left(\theta_{0}, r_{0}\right)
$$

where $H_{2}\left(\theta_{0}, r_{0}\right)=H\left(\theta_{0}, r_{0}\right)+H\left(\theta_{1}, r_{1}\right)+\frac{1}{r_{1}} \mu_{1}\left(\theta_{1}\right)-\frac{1}{r_{0}} \mu_{1}\left(\theta_{0}+2 \alpha \pi\right)$. Obviously, we have that

$$
\lim _{r_{0} \rightarrow+\infty} r_{0}\left|H_{2}\left(\theta_{0}, r_{0}\right)\right|=0
$$

On the other hand, since

$$
\mu_{2}\left(\theta_{1}\right)=\mu_{2}\left(\theta_{0}+2 \alpha \pi+\frac{1}{r_{0}} \mu_{1}\left(\theta_{0}\right)+H\left(\theta_{0}, r_{0}\right)\right)=\mu_{2}\left(\theta_{0}+2 \alpha \pi\right)+O\left(\frac{1}{r_{0}}\right),
$$

we get that

$$
r_{2}=r_{0}+\mu_{2}\left(\theta_{0}\right)+\mu_{2}\left(\theta_{0}+2 \alpha \pi\right)+G_{2}\left(\theta_{0}, r_{0}\right),
$$


where $G_{2}\left(\theta_{0}, r_{0}\right)=G\left(\theta_{0}, r_{0}\right)+G\left(\theta_{1}, r_{1}\right)+\mu_{2}\left(\theta_{1}\right)-\mu_{2}\left(\theta_{0}+2 \alpha \pi\right)$. It is easy to check that

$$
\lim _{r_{0} \rightarrow+\infty}\left|G_{2}\left(\theta_{0}, r_{0}\right)\right|=0 .
$$

Inductively, we have that

$$
\left\{\begin{array}{l}
\theta_{n}=\theta_{0}+2 n \alpha \pi+\frac{1}{r_{0}} \sum_{i=0}^{i=n-1} \mu_{1}\left(\theta_{0}+2 i \alpha \pi\right)+H_{n}\left(\theta_{0}, r_{0}\right) \\
r_{n}=r_{0}+\sum_{i=0}^{i=n-1} \mu_{2}\left(\theta_{0}+2 i \alpha \pi\right)+G_{n}\left(\theta_{0}, r_{0}\right)
\end{array}\right.
$$

where $H_{n}\left(\theta_{0}, r_{0}\right)$ and $G_{n}\left(\theta_{0}, r_{0}\right)$ satisfy

$$
r_{0}\left|H_{n}\left(\theta_{0}, r_{0}\right)\right|+\left|G_{n}\left(\theta_{0}, r_{0}\right)\right| \rightarrow 0 \text { as } r_{0} \rightarrow+\infty .
$$

Next, we define a transformation $T: S^{1} \rightarrow S^{1}, T(\theta)=\theta+2 \alpha \pi$. Since $\alpha$ is an irrational number, $T$ is ergodic. By the Birkhoff Ergodic Theorem [13] we get that

$$
\lim _{n \rightarrow+\infty} \frac{1}{n} \sum_{i=0}^{i=n-1} \mu_{2}(\theta+2 i \alpha \pi)=\lim _{n \rightarrow+\infty} \frac{1}{n} \sum_{i=0}^{i=n-1} \mu_{2}\left(T^{i} \theta\right)=\frac{1}{2 \pi} \int_{0}^{2 \pi} \mu_{2}(\theta) d \theta>0
$$

for almost every $\theta \in S^{1}$. Since $\mu_{2}$ is continuous and $S^{1}$ is compact, we can further obtain that

$$
\lim _{n \rightarrow+\infty} \frac{1}{n} \sum_{i=0}^{i=n-1} \mu_{2}(\theta+2 i \alpha \pi)=\frac{1}{2 \pi} \int_{0}^{2 \pi} \mu_{2}(\theta) d \theta>0
$$

uniformly for every $\theta \in S^{1}$. Therefore, there exist a positive integer $m>>1$ and a constant $c>0$ such that

$$
\frac{1}{m} \sum_{i=0}^{i=m-1} \mu_{2}\left(\theta_{0}+2 i \alpha \pi\right) \geq c>0
$$

for all $\theta_{0} \in S^{1}$. Recalling that $\lim _{r_{0} \rightarrow+\infty} G_{m}\left(\theta_{0}, r_{0}\right)=0$, we have that there exists a constant $R_{0}>\sigma$ such that for $r_{0} \geq R_{0},\left|G_{m}\left(\theta_{0}, r_{0}\right)\right| \leq c$. Then for $r_{0} \geq R_{0}$, we get that

$$
\begin{aligned}
r_{m}=r_{0}+m \cdot \frac{1}{m} \sum_{i=0}^{i=m-1} \mu_{2}\left(\theta_{0}+2 i \alpha \pi\right)+G_{m}\left(\theta_{0}, r_{0}\right) & \geq r_{0}+m c+G_{m}\left(\theta_{0}, r_{0}\right) \\
& \geq r_{0}+(m-1) c .
\end{aligned}
$$

Meanwhile, we have that

$$
\begin{aligned}
r_{2 m} & =r_{m}+m \cdot \frac{1}{m} \sum_{i=0}^{i=m-1} \mu_{2}\left(\theta_{m}+2 i \alpha \pi\right)+G_{m}\left(\theta_{m}, r_{m}\right) \\
& \geq r_{m}+m c+G_{m}\left(\theta_{m}, r_{m}\right) \geq r_{m}+(m-1) c \geq r_{0}+2(m-1) c .
\end{aligned}
$$

Inductively, we have that

$$
\begin{aligned}
r_{k m} & =r_{(k-1) m}+m \cdot \frac{1}{m} \sum_{i=0}^{i=m-1} \mu_{2}\left(\theta_{(k-1) m}+2 i \alpha \pi\right)+G_{m}\left(\theta_{(k-1) m}, r_{(k-1) m}\right) \\
& \geq r_{(k-1) m}+m c+G_{m}\left(\theta_{(k-1) m}, r_{(k-1) m}\right) \geq r_{0}+k(m-1) c .
\end{aligned}
$$

Therefore, we get that

$$
\lim _{k \rightarrow+\infty} r_{k m}=+\infty
$$


Because $\mu_{2}(\theta)$ is continuous and $\lim _{r_{0} \rightarrow+\infty} G\left(\theta_{0}, r_{0}\right)=0$, there exists a constant $d>0$ such that

$$
\left|\mu_{2}\left(\theta_{0}\right)+G\left(\theta_{0}, r_{0}\right)\right| \leq d,
$$

for $\theta_{0} \in S^{1}$ and $r_{0}>\sigma$. From

$r_{(k m+i)}=r_{(k m+i-1)}+\mu_{2}\left(\theta_{(k m+i-1)}\right)+G\left(\theta_{(k m+i-1)}, r_{(k m+i-1)}\right), i=1, \cdots, m-1$, we get that

$$
\left|r_{(k m+i)}-r_{(k m+i-1)}\right| \leq d, \quad i=1, \cdots, m-1 .
$$

Consequently, we have that

$$
\left|r_{(k m+i)}-r_{k m}\right| \leq i d, \quad i=1, \cdots, m-1 .
$$

From (2.4) and (2.5) we know that

$$
\lim _{n \rightarrow+\infty} r_{n}=+\infty
$$

Proposition 2.2. Assume that conditions (2.2), (2.3) hold and

$$
\int_{0}^{2 \pi} \mu_{2}(\theta) d \theta<0 \text {. }
$$

Then there exists $R_{0}>\sigma$ such that if $r_{0} \geq R_{0}$, the orbit $\left\{\left(\theta_{n}, r_{n}\right)\right\}$ satisfies

$$
\lim _{n \rightarrow-\infty} r_{n}=+\infty \text {. }
$$

The proof of Proposition 2.2 is identical to the proof of Proposition 2.1. We only give some explanations. At first, from $(2.2),(2.3)$ we know that $\mathcal{P}\left(E_{\sigma}\right)$ contains a neighborhood of infinity. Next, by using the inductive method, we can also obtain that

$$
\left\{\begin{array}{l}
\theta_{-n}=\theta_{0}-2 n \alpha \pi-\frac{1}{r_{0}} \sum_{i=0}^{i=n-1} \mu_{1}\left(\theta_{0}-2 i \alpha \pi\right)-H_{-n}\left(\theta_{0}, r_{0}\right), \\
r_{-n}=r_{0}-\sum_{i=0}^{i=n-1} \mu_{2}\left(\theta_{0}-2 i \alpha \pi\right)-G_{-n}\left(\theta_{0}, r_{0}\right),
\end{array}\right.
$$

where $H_{-n}\left(\theta_{0}, r_{0}\right)$ and $G_{-n}\left(\theta_{0}, r_{0}\right)$ satisfy

$$
r_{0}\left|H_{-n}\left(\theta_{0}, r_{0}\right)\right|+\left|G_{-n}\left(\theta_{0}, r_{0}\right)\right| \rightarrow 0 \text { as } r_{0} \rightarrow+\infty .
$$

Thus, by applying the same ideas in proving Proposition 2.1, we can prove that the conclusion of Proposition 2.2 holds.

\section{ACtion AND ANGLE VARIABles}

At first, we consider the piecewise linear equation

$$
x^{\prime \prime}+a x^{+}-b x^{-}=0
$$

and denote by $C(t)$ the solution of (3.1) satisfying the initial condition $x(0)=$ $1, x^{\prime}(0)=0$. It is a periodic function with period

$$
\tau=\frac{\pi}{\sqrt{a}}+\frac{\pi}{\sqrt{b}}
$$

and can be expressed by

$$
C(t)=\left\{\begin{array}{l}
\cos \sqrt{a} t, \quad 0 \leq|t| \leq \frac{\pi}{2 \sqrt{a}} \\
-\sqrt{\frac{a}{b}} \sin \sqrt{b}\left(t-\frac{\pi}{2 \sqrt{a}}\right), \quad \frac{\pi}{2 \sqrt{a}} \leq|t| \leq \frac{\tau}{2} .
\end{array}\right.
$$


The derivative of $C(t)$ will be denoted by $S(t)=C^{\prime}(t)$. Obviously, $C(t)$ and $S(t)$ satisfy the following properties,

(i) $C(t+\tau)=C(t), S(t+\tau)=S(t)$ and $C(0)=1, S(0)=0$.

(ii) $C(t) \in C^{2}(\mathbb{R}), S(t) \in C^{1}(\mathbb{R})$.

(iii) $C^{\prime}(t)=S(t), S^{\prime}(t)=-\left(a C^{+}(t)-b C^{-}(t)\right)$.

(iv) $S(t)^{2}+a C^{+}(t)^{2}+b C^{-}(t)^{2}=a, \quad \forall t \in \mathbb{R}$.

Define the mapping

$$
\Phi: \quad(\theta, I) \in S^{1} \times(0,+\infty) \rightarrow(x, y) \in \mathbb{R}^{2} \backslash\{0\}
$$

with

$$
x=\gamma I^{\frac{1}{2}} C\left(\frac{\theta}{\omega}\right), \quad y=\gamma I^{\frac{1}{2}} S\left(\frac{\theta}{\omega}\right),
$$

with $\omega=\frac{2 \pi}{\tau}, \gamma=\sqrt{\frac{2 \omega}{a}}$. It is easy to check that $\Phi$ is an area-preserving $C^{1}$ diffeomorphism.

Now, we deal with Eq. (1.1). Consider the equivalent system of Eq. (1.1)

$$
x^{\prime}=y-F(x), \quad y^{\prime}=-\left(a x^{+}-b x^{-}\right)+p(t) .
$$

Under the transformation $\Phi$, Eq. (3.2) becomes

$$
\left\{\begin{array}{l}
\frac{d \theta}{d t}=\omega+\frac{\gamma}{2} I^{-\frac{1}{2}} F\left(\gamma I^{\frac{1}{2}} C\left(\frac{\theta}{\omega}\right)\right) S\left(\frac{\theta}{\omega}\right)-\frac{\gamma}{2} I^{-\frac{1}{2}} p(t) C\left(\frac{\theta}{\omega}\right), \\
\frac{d I}{d t}=\frac{2}{a \gamma} I^{\frac{1}{2}}\left[-a C^{+}\left(\frac{\theta}{\omega}\right)+b C^{-}\left(\frac{\theta}{\omega}\right)\right] F\left(\gamma I^{\frac{1}{2}} C\left(\frac{\theta}{\omega}\right)\right)+\frac{2}{a \gamma} I^{\frac{1}{2}} p(t) S\left(\frac{\theta}{\omega}\right) .
\end{array}\right.
$$

Denote by $\left(\theta\left(t ; \theta_{0}, I_{0}\right), I\left(t ; \theta_{0}, I_{0}\right)\right)$ the solution of $(3.3)$ satisfying an initial condition $\theta(0)=\theta_{0}, I(0)=I_{0}$. If $F(x)$ is bounded, then for large values of $I_{0}$, this solution is defined for all $t \in[0,2 \pi]$. Thus we can define the Poincaré mapping

$$
\theta_{1}=\theta\left(2 \pi ; \theta_{0}, I_{0}\right), \quad I_{1}=I\left(2 \pi ; \theta_{0}, I_{0}\right) .
$$

From the second equality of $(3.3)$ we get that

$$
\frac{d I^{\frac{1}{2}}}{d t}=\frac{4}{a \gamma}\left[\left(-a C^{+}\left(\frac{\theta}{\omega}\right)+b C^{-}\left(\frac{\theta}{\omega}\right)\right) F\left(\gamma I^{\frac{1}{2}} C\left(\frac{\theta}{\omega}\right)\right)+p(t) S\left(\frac{\theta}{\omega}\right)\right] .
$$

It follows from (3.4) that

$$
I(t)^{\frac{1}{2}}=I_{0}^{\frac{1}{2}}+O(1), t \in[0,2 \pi], I_{0} \rightarrow+\infty .
$$

Furthermore, we have that

$$
I(t)^{-\frac{1}{2}}=I_{0}^{-\frac{1}{2}}+O\left(I_{0}^{-1}\right), t \in[0,2 \pi], I_{0} \rightarrow+\infty .
$$

From (3.5) and the first equality of (3.3) we know that

$$
\frac{d \theta}{d t}=\omega+O\left(I_{0}^{-\frac{1}{2}}\right)
$$

Consequently,

$$
\theta(t)=\theta_{0}+\omega t+O\left(I_{0}^{-\frac{1}{2}}\right), t \in[0,2 \pi],
$$

which, together with (3.4), yields

$$
\begin{aligned}
\frac{d I^{\frac{1}{2}}}{d t}= & \frac{4}{a \gamma}\left[\left(-a C^{+}\left(t+\frac{\theta_{0}}{\omega}\right)+b C^{-}\left(t+\frac{\theta_{0}}{\omega}\right)\right) F\left(\gamma I_{0}^{\frac{1}{2}} C\left(t+\frac{\theta_{0}}{\omega}\right)+O(1)\right)+p(t) S\left(t+\frac{\theta_{0}}{\omega}\right)\right] \\
& +O\left(I_{0}^{-\frac{1}{2}}\right) .
\end{aligned}
$$


An integration shows that

$$
\begin{aligned}
I_{1}^{\frac{1}{2}}= & I_{0}^{\frac{1}{2}}+\frac{4}{a \gamma} \int_{0}^{2 \pi}\left(-a C^{+}\left(t+\frac{\theta_{0}}{\omega}\right)+b C^{-}\left(t+\frac{\theta_{0}}{\omega}\right)\right) F\left(\gamma I_{0}^{\frac{1}{2}} C\left(t+\frac{\theta_{0}}{\omega}\right)+O(1)\right) d t \\
& +\frac{4}{a \gamma} \int_{0}^{2 \pi} p(t) S\left(t+\frac{\theta_{0}}{\omega}\right) d t+O\left(I_{0}^{-\frac{1}{2}}\right) .
\end{aligned}
$$

Similarly, substituting (3.6) in the first equality of (3.3), we obtain that for $t \in$ $[0,2 \pi]$

$\frac{d \theta}{d t}=\omega+\frac{\gamma}{2} I_{0}^{-\frac{1}{2}} F\left(\gamma I_{0}^{\frac{1}{2}} C\left(t+\frac{\theta_{0}}{\omega}\right)+O(1)\right) S\left(t+\frac{\theta_{0}}{\omega}\right)-\frac{\gamma}{2} I_{0}^{-\frac{1}{2}} p(t) C\left(t+\frac{\theta_{0}}{\omega}\right)+O\left(I_{0}^{-1}\right)$.

Therefore, we have that

$$
\begin{aligned}
\theta_{1}= & \theta_{0}+2 \pi \omega+\frac{\gamma}{2} I_{0}^{-\frac{1}{2}} \int_{0}^{2 \pi} F\left(\gamma I_{0}^{\frac{1}{2}} C\left(t+\frac{\theta_{0}}{\omega}\right)+O(1)\right) S\left(t+\frac{\theta_{0}}{\omega}\right) d t \\
& -\frac{a \gamma}{2} I_{0}^{-\frac{1}{2}} \int_{0}^{2 \pi} p(t) C\left(t+\frac{\theta_{0}}{\omega}\right) d t+O\left(I_{0}^{-1}\right) .
\end{aligned}
$$

Set $r=I^{1 / 2}$. Then we get

$$
\left\{\begin{aligned}
\theta_{1}= & \theta_{0}+2 \pi \omega+\frac{\gamma}{2} r_{0}^{-1} \int_{0}^{2 \pi} F\left(\gamma r_{0} C\left(t+\frac{\theta_{0}}{\omega}\right)+O(1)\right) S\left(t+\frac{\theta_{0}}{\omega}\right) d t \\
& -\frac{\gamma}{2} r_{0}^{-1} \int_{0}^{2 \pi} p(t) C\left(t+\frac{\theta_{0}}{\omega}\right) d t+O\left(r_{0}^{-2}\right) \\
r_{1}= & r_{0}+\frac{4}{a \gamma} \int_{0}^{2 \pi}\left(-a C^{+}\left(t+\frac{\theta_{0}}{\omega}\right)+b C^{-}\left(t+\frac{\theta_{0}}{\omega}\right)\right) F\left(\gamma r_{0} C\left(t+\frac{\theta_{0}}{\omega}\right)+O(1)\right) d t \\
& +\frac{4}{a \gamma} \int_{0}^{2 \pi} p(t) S\left(t+\frac{\theta_{0}}{\omega}\right) d t+O\left(r_{0}^{-1}\right) .
\end{aligned}\right.
$$

Write

$$
\begin{aligned}
& \psi_{1}\left(\theta_{0}, r_{0}\right)=\int_{0}^{2 \pi} F\left(\gamma r_{0} C\left(t+\frac{\theta_{0}}{\omega}\right)+O(1)\right) S\left(t+\frac{\theta_{0}}{\omega}\right) d t, \\
& \psi_{2}\left(\theta_{0}, r_{0}\right)=\int_{0}^{2 \pi}\left(-a C^{+}\left(t+\frac{\theta_{0}}{\omega}\right)+b C^{-}\left(t+\frac{\theta_{0}}{\omega}\right)\right) F\left(\gamma r_{0} C\left(t+\frac{\theta_{0}}{\omega}\right)+O(1)\right) d t, \\
& \psi_{3}\left(\theta_{0}\right)=\int_{0}^{2 \pi} p(t) C\left(t+\frac{\theta_{0}}{\omega}\right) d t, \\
& \psi_{4}\left(\theta_{0}\right)=\int_{0}^{2 \pi} p(t) S\left(t+\frac{\theta_{0}}{\omega}\right) d t .
\end{aligned}
$$

Lemma 1. Assume that the limits $\lim _{x \rightarrow+\infty} F(x)=F(+\infty), \lim _{x \rightarrow-\infty} F(x)=$ $F(-\infty)$ exist and are finite. Then, for $r_{0} \rightarrow+\infty$,

$$
\begin{aligned}
& \psi_{1}\left(\theta_{0}, r_{0}\right)=F(+\infty) \int_{J_{1}} S\left(t+\frac{\theta_{0}}{\omega}\right) d t+F(-\infty) \int_{J_{2}} S\left(t+\frac{\theta_{0}}{\omega}\right) d t+o(1), \\
& \psi_{2}\left(\theta_{0}, r_{0}\right)=-a F(+\infty) \int_{J_{1}} C^{+}\left(t+\frac{\theta_{0}}{\omega}\right) d t+b F(-\infty) \int_{J_{2}} C^{-}\left(t+\frac{\theta_{0}}{\omega}\right) d t+o(1),
\end{aligned}
$$

where $J_{1}=\left\{t: t \in(0,2 \pi), C\left(t+\frac{\theta_{0}}{\omega}\right) \geq 0\right\}, J_{2}=\left\{t: t \in(0,2 \pi), C\left(t+\frac{\theta_{0}}{\omega}\right) \leq 0\right\}$.

Proof. We only check that

$$
\lim _{r_{0} \rightarrow+\infty} \int_{J_{1}} F\left(\gamma r_{0} C\left(t+\frac{\theta_{0}}{\omega}\right)+O(1)\right) S\left(t+\frac{\theta_{0}}{\omega}\right) d t=F(+\infty) \int_{J_{1}} S\left(t+\frac{\theta_{0}}{\omega}\right) d t .
$$


From $\lim _{x \rightarrow+\infty} F(x)=F(+\infty)$ we have that, for any sufficiently small $\eta>0$,

$$
\lim _{r_{0} \rightarrow+\infty} \int_{J_{11}} F\left(\gamma r_{0} C\left(t+\frac{\theta_{0}}{\omega}\right)+O(1)\right) S\left(t+\frac{\theta_{0}}{\omega}\right) d t=F(+\infty) \int_{J_{11}} S\left(t+\frac{\theta_{0}}{\omega}\right) d t,
$$

with $J_{11}=\left\{t: t \in(0,2 \pi), C\left(t+\frac{\theta_{0}}{\omega}\right) \geq \eta\right\}$. On the other hand, it is easy to see that

$$
\lim _{\eta \rightarrow 0^{+}} \int_{J_{12}} F\left(\gamma r_{0} C\left(t+\frac{\theta_{0}}{\omega}\right)+O(1)\right) S\left(t+\frac{\theta_{0}}{\omega}\right) d t=0, \quad \lim _{\eta \rightarrow 0^{+}} \int_{J_{12}} S\left(t+\frac{\theta_{0}}{\omega}\right) d t=0,
$$

where $J_{12}=\left\{t: t \in(0,2 \pi), 0 \leq C\left(t+\frac{\theta_{0}}{\omega}\right) \leq \eta\right\}$. Thus we get the conclusion.

Lemma 2. $\int_{0}^{2 \pi} \psi_{4}\left(\theta_{0}\right) d \theta_{0}=0$.

Proof. Since $\psi_{4}\left(\theta_{0}\right)=\psi_{3}^{\prime}\left(\theta_{0}\right)$ and $\psi_{4}\left(\theta_{0}\right), \psi_{3}\left(\theta_{0}\right)$ are $2 \pi$-periodic functions, we have $\int_{0}^{2 \pi} \psi_{4}\left(\theta_{0}\right) d \theta_{0}=0$.

Now, we prove Theorem 1 . The proof of Theorem 2 can be treated similarly.

Proof of Theorem 1. Consider the Poincaré mapping $P:\left(\theta_{0}, r_{0}\right) \rightarrow\left(\theta_{1}, r_{1}\right)$. From Lemma 1 we know that $P$ can be expressed in the form:

$$
\left\{\begin{array}{l}
\theta_{1}=\theta_{0}+2 \pi \omega+r_{0}^{-1} \mu_{1}\left(\theta_{0}\right)+H\left(\theta_{0}, r_{0}\right), \\
r_{1}=r_{0}+\mu_{2}\left(\theta_{0}\right)+G\left(\theta_{0}, r_{0}\right),
\end{array}\right.
$$

where $H, G$ are continuous functions and satisfy

$$
H\left(\theta_{0}, r_{0}\right)=o\left(\frac{1}{r_{0}}\right), \quad G\left(\theta_{0}, r_{0}\right)=o(1) \text { as } r_{0} \rightarrow+\infty
$$

and $\mu_{1}\left(\theta_{0}\right)=\frac{\gamma}{2}\left[\phi_{1}\left(\theta_{0}\right)-\psi_{3}\left(\theta_{0}\right)\right], \mu_{2}\left(\theta_{0}\right)=\frac{4}{a \gamma}\left[\phi_{2}\left(\theta_{0}\right)+\psi_{4}\left(\theta_{0}\right)\right]$ with

$$
\begin{gathered}
\phi_{1}\left(\theta_{0}\right)=F(+\infty) \int_{J_{1}} S\left(t+\frac{\theta_{0}}{\omega}\right) d t+F(-\infty) \int_{J_{2}} S\left(t+\frac{\theta_{0}}{\omega}\right) d t, \\
\phi_{2}\left(\theta_{0}\right)=-a F(+\infty) \int_{J_{1}} C^{+}\left(t+\frac{\theta_{0}}{\omega}\right) d t+b F(-\infty) \int_{J_{2}} C^{-}\left(t+\frac{\theta_{0}}{\omega}\right) d t,
\end{gathered}
$$

where $J_{1}$ and $J_{2}$ are defined in Lemma 1 . Clearly, $\mu_{1}, \mu_{2}: S^{1} \rightarrow S^{1}$ are Lipschitz continuous. Since $1 / \sqrt{a}+1 / \sqrt{b} \in \mathbb{R} \backslash \mathbb{Q}$ and $\omega=2 \pi / \tau, \tau=\pi / \sqrt{a}+\pi / \sqrt{b}$, we have that $\omega$ is an irrational number. On the other hand, it follows from $F(+\infty)<0<$ $F(-\infty)$ that $\phi_{2}\left(\theta_{0}\right)>0$ for $\theta_{0} \in S^{1}$. Therefore, from Lemma 2 we get that

$$
\int_{0}^{2 \pi} \mu_{2}\left(\theta_{0}\right) d \theta_{0}=\frac{4}{a \gamma} \int_{0}^{2 \pi} \phi_{2}\left(\theta_{0}\right) d \theta_{0}>0 .
$$

Applying the result of Proposition 2.1, we obtain the conclusion of Theorem 1.

\section{REFERENCES}

[1] N. Dancer, On the Dirichlet problem for weakly nonlinear elliptic partial differential equations, Proc. Roy. Soc. Edinburg Scct, A(76)1977, 283-300. MR 82i:35063 MR 58:17506

[2] N. Dancer, Boundary-value problems for weakly nonlinear ordinary differential equations, Bull. Austral. Math. Soc., 15(1976), 321-328. MR 55:3389

[3] S. Fučik, Solvability of nonlinear equations and boundary value problems, Reidel Dordrecht, 1980. MR 83c: 47079

[4] P. Drabek and S. Invernizzi, On the periodic boundary value theorem for forced Duffing equation with jumping nonlinearity, Nonlinear Anal, 10(1986), 643-650. MR 87j:34077 
[5] C. Fabry, Landesman-lazer conditions for periodic boundary value problems wih asymmetric nonlinearities, J. Differential Equations, 116(1995), 405-418. MR 96c:34033

[6] C. Fabry and A. Fonda, Nonlinear resonance in asymmetric oscillators, J. Differential Equations, 147(1998), 58-78. MR 99d:34070

[7] A. C. Lazer and D. E. Leach, Bounded perturbations of forced harmonic oscillations at resonance, Ann. Mat. Pura. Appl., (82)1969, 49-68. MR 40:2972

[8] T. Ding, Nonlinear oscillations at a point of resonance, Scientia Sinica(series A), (8)1982, 918-931. MR 84c:34058

[9] B. Liu, Boundedness in nonlinear oscillations at resonance, J. Differential Equations, 153(1999), 142-172. MR 2000d:34075

[10] R. Ortega, Asymmetric oscillators and twist mappings, J. London Math. Soc., 53(1996), 325-342. MR 96k:34093

[11] J. M. Alonso and R. Ortega, Unbounded solutions of semilinear equations at resonance, Nonlinearity, 9(1996), 1099-1111. MR 97m:35155

[12] J. M. Alonso and R. Ortega, Roots of unity and unbounded motions of an asymmetric oscillator, J. Differential Equations, 143(1998), 201-220. MR 99a:34102

[13] P. Walters, An introduction to ergodic theory, Springer-Verlag, 1982. MR 84e:28017

Department of Mathematics, Capital Normal University, Beijing 100037, People's RePublic OF China

E-mail address: zhwang@mail.cnu.edu.cn 